\title{
Anomalous temperature dependence of the first diffraction peak in vitreous boron trioxide
}

\author{
F. J. Bermejo, J. Dawidowski, and R. Fernández-Perea \\ Instituto de Estructura de la Materia, Consejo Superior de Investigaciones Científicas, Serrano 123, Madrid E-28006, Spain \\ J. L. Martínez \\ Instituto de Ciencia de Materiales, Consejo Superior de Investigaciones Científicas, E-28040 Cantoblanco, Spain
}

(Received 9 February 1996)

\begin{abstract}
The temperature dependence of the parameters characterizing the first peak in the $S(Q)$ and $S(Q, E=0)$ diffraction patterns of vitreous boron trioxide is considered in some detail. The analysis of the experimental spectra is aided by results derived from molecular-dynamics simulations, which enable us to isolate the most relevant features driving the variation with temperature of both structure factors. The relevance of the present results regarding some recent phenomenological approaches developed towards the understanding of the dynamics of glasses at intermediate temperatures is finally discussed. [S0163-1829(96)00325-6]
\end{abstract}

\section{INTRODUCTION}

The purpose of this work is to help to clarify the origin of the apparently anomalous behavior with temperature exhibited by the first intense peak in the $S(Q)$ static structure factor (very often referred to as the first sharp diffraction peak or FSDP), of a strong glass, vitreous boron trioxide, which appears in the diffraction pattern at wave numbers of $\approx 1.6 \AA^{-1}$. The relevance of such a study stems from current attempts ${ }^{1}$ assigning a special significance to the parameters characterizing such a peak (i.e., its location $Q_{p}$, width $\Delta_{Q}$ and intensity $I_{Q}$, which are often interpreted as evidences of the existence of positional ${ }^{2}$ or chemical ${ }^{3}$ medium-range order (MRO), that is the presence of structural regularities involving distances beyond some 5-10 $\AA$ characteristic of the location of second-nearest neighbors. The emphasis will be put into the exploration of the temperature dependence of the parameters which describe the first peak in the total, $S(Q)$ (i.e., frequency-integrated) and elastic $S(Q, E=0)$ structure factors of vitreous $\mathrm{B}_{2} \mathrm{O}_{3}$ as measured using cold neutron spectroscopy. The distinction between total and elastic components is by no means trivial as demonstrated experimentally by previous results regarding glassy selenium $g$-Se, ${ }^{4}$ or by theoretical attempts of a phenomenological nature which try to rationalize the most relevant features of the glassy dynamics in terms of simplified constructs such as those portraying it in terms of particle motions within onedimensional anharmonic potentials. ${ }^{5}$ In fact, as shown in recent work on $g-\mathrm{Se}^{4}$ the interpretation of realspace parameters, such as $R=2 \pi / Q_{p}$ and $R_{c}=2 \pi / \Delta_{Q}$, in terms of a characteristic distance and a correlation length could only be pursued if the former corresponds to a $Q_{p}$ defining the maxima of the elastic quantity whereas the latter regards the one specifying the peak width of $S(Q)$. This comes as a consequence of the increasingly differing shapes of $S(Q)$ and $S(Q, E=0)$ as the temperature is raised, which leads to rather disparate behaviors in the temperature dependences of $R$ and $R_{c}$ derived from the static or elastic component. Furthermore, a clear distinction between the two structure factors is also required to avoid controversies like those produced in the recent past ${ }^{6}$ regarding the temperature dependence of the FSDP in vitreous silica, where different conclusions regarding the temperature dependence of the FSDP were reached depending upon the way of measurement of such a feature [i.e. total diffraction or integrals over the $S(Q, E)$ structure factor measured in inelastic neutron experiments. $\left.{ }^{6}\right]$

Although the subject of the existence of MRO has been debated a number of times, ${ }^{2,3}$ the presence of stereochemical regularities up to some $10-20 \AA$ in a good number of glasses, which in some cases persist well within the molten state, is now a well established fact. The discussion is therefore centered on the microscopic origin (if any) of the FSDP as well as the mechanisms driving its dependence with temperature, which in some cases as mentioned some time ago, ${ }^{7}$ evidences an anomalous behavior, reminiscent in some aspects of that shown by liquid ${ }^{4} \mathrm{He}$ below the $\lambda$ transition. ${ }^{8}$ To explain such a behavior in systems where quantum effects can be safely disregarded some arguments have been brought forward. Between those, the one due to Busse, ${ }^{7}$ later considered in detail by Vashista et al. ${ }^{9}$ merits some detailed consideration since it qualitatively accounts for the observed trends. Such an attempt tries to explain the increase in height of the FSDP as the temperature is raised as arising from a decrease in "frustration" (i.e., a competition between the tendency of the stereochemical units to form structures compatible with those seen in the crystals and the available free space for such rearrangements) as the density is decreased due to thermal expansion. Our contribution in this respect will be focused towards the separation of those effects due to a change in density from those originated by an increase with temperature of thermal motions. As it will be shown below, the concurrent use of neutron scattering and computer simulations enabled us to unveil the effects of a number of dynamic phenomena which certainly contribute significantly to relaxational (i.e., nonvibrational) processes within the glass phase which should also be considered in some detail for a more realistic description of the glassy dynamics.

The present work also aims to extend and rationalize some of the available results mostly derived from diffraction means at high temperatures ${ }^{10,11}$ concerning the density and thermal treatment dependence of several features appearing 
in the neutron or x-ray-diffraction patterns, by means of measurements performed at temperatures well within the glass phase. It is precisely at temperatures well below ambient where the characteristic anomalies of the glassy dynamics such as the excess $C(T) / T^{3}$ specific heat and large negative Grüneisen parameters ${ }^{12}(5-30 \mathrm{~K})$, the large peak in the internal friction ${ }^{13}(\approx 100 \mathrm{~K})$ or the noticeable change in the depolarization ratio of some Raman peaks ${ }^{14}$ manifest themselves.

\section{EXPERIMENTAL AND CALCULATIONAL DETAILS}

From an experimental standpoint, the data presented here correspond to a set measured using the TAS7 coldneutron triple-axis spectrometer located at one of the beam tubes of the DR3 reactor of the Risoe National Laboratory (Denmark). The sample which was made of vitreous ${ }^{11} \mathrm{~B}_{2}^{\text {nat }} \mathrm{O}_{3}$ with a stated ${ }^{11} \mathrm{~B}$ enrichment of $98 \%$, was purchased from the Commisariat de l'Energie Atomique (France). Sample purity was checked by means of infrared spectroscopy. Particular attention was paid to the quantitative analysis of the water content, since the effect of bulk water is known to affect drastically most of the mechanical properties of the solid. ${ }^{13}$ Up to experimental precision, no signal distinguishable from the background was detected at frequencies characteristic of the $\mathrm{O}-\mathrm{H}$ stretching vibration, so that for most purposes the sample may be considered as dry. It was then sealed within an aluminum plate of thickness $3.8 \mathrm{~mm}$ which enabled a transmission of some $80 \%$. Two sets of measurements were carried with [for the $S(Q, E=0)$ ] or without [for $S(Q)$ ] energy analysis of the scattered neutrons, both of them employing an incident energy $E_{0}=5 \mathrm{meV}$, which enabled a resolution in energy transfers [i.e., for the determination of the $S(Q, E=0)$ elastic component] of some $0.09 \mathrm{meV}$ full width at half maximum. The measurements were carried out at 23 different temperatures ranging from 5 up to $300 \mathrm{~K}$. The measured cross sections were corrected for the relatively strong absorption effects using the program CORRECT. ${ }^{15} \mathrm{~A}$ correction for the resolution effects in energy transfers was also applied to the $S(Q, E=0)$ structure factors, by adjusting the $S(Q, E=0)$ value to the corresponding for $S(Q)$ at the lowest available temperature and $Q$ value. On the other hand and to help with the analysis of experimental spectra, some results from molecular-dynamics (MD) simulations for a realistic model of vitreous $\mathrm{B}_{2} \mathrm{O}_{3}$ (see Ref. 16 for details), were also brought forward. Such additional pieces of information enabled us to access some quantities difficult to reach by experimental means such as the $S_{\alpha \beta}(Q, E)$ partial dynamic structure factors.

In addition to results given in Ref. 16 several other quantities have been calculated to aid with the analysis of the experimental results. These refer to a more detailed look into the atomic dynamics at high $(300 \mathrm{~K})$ temperatures where we have followed the changes in the bonding structure during 65 ps. Also several simulations using a larger box containing 2000 atoms and a density of $1.82 \mathrm{~g} \mathrm{~cm}^{-3}$ were performed for both low and high temperatures, thus enabling the exploration of correlations extending up to some $15 \AA$. In order to get some estimates for the total atomic displacement at both sides of the glass transition, (the calorimetric transition is located at $T_{g} \approx 533 \mathrm{~K}$ ) several simulation runs comprising temperatures from 100 to $2000 \mathrm{~K}$ were carried out, from where estimates of the averaged meansquared atomic displacements were obtained.

\section{RESULTS AND DISCUSSION}

\section{A. Structure factors}

Some experimental results for $S(Q)$ and $S(Q, E=0)$ regarding two extreme temperatures as well as the temperature dependence of the parameters used to describe those peaks as Gaussians are shown in Fig. 1. The most remarkable difference between both sets of results regards the somewhat narrower shape of peaks in the elastic, and to a far lesser extent in the static, quantities at high temperature, as well as the noticeable departure of the static quantity from the one measured using total scattering (diffraction) techniques. ${ }^{17}$ The counterintuitive result of peaks in $S(Q, E=0)$ becoming narrower as the temperature increases is also borne out by results calculated by computer simulation as data plotted in Fig. 2 demonstrates. In fact, the width of the peak in both, the calculated $S(Q)$ and the present experiment becomes substantially broader than that of Johnson et al. ${ }^{17}$ as well as the peak maxima is shifted to higher $Q$ values than in that work. The upper-left frame shows the $S(Q)$ as calculated by integration of the total $I(Q, t)$ (symbols) as well as from the $g(r)$ (lines) at 0.1 and $300 \mathrm{~K}$. The difference of these two curves is shown in the inset, where small although systematic differences in the structure factors are observed. In the lower-left frame, the elastic peak for both temperatures are shown. As expected, and in agreement with data measured at higher temperatures $\left(121 \mathrm{~K}\right.$ upwards $\left.{ }^{10}\right)$, the peak maxima of the low-temperature experimental elastic structure factors get shifted towards smaller wave numbers by some $2.6 \times 10^{4}$ $\mathrm{K}^{-1}$, whereas the static follows the same behavior although far less markedly (i.e., by some $3.3 \times 10^{5} \mathrm{~K}^{-1}$ ), both behaviors being qualitatively reproduced by the simulation results. Such findings seem to lend additional support to the hypothesis of assigning to $2 \pi / Q_{p}$ the meaning of a characteristic distance which, simply due to a density effect, increases with increasing temperature. Such values for the temperature coefficient can be compared with those for the macroscopic thermal-expansion coefficient which above $4 \mathrm{~K}$ is always positive $\mathrm{e}^{12}$ with values of $\chi=4.6 \times 10^{5} \mathrm{~K}^{-1}$ at $T \approx 280 \mathrm{~K}$.

Far more remarkable is the fact that the observed trends regarding the peak widths are also followed by the three $S_{\alpha \beta}(Q, E=0), \alpha \beta=\mathrm{OO}, \mathrm{BB}, \mathrm{BO}$ different partial structure factors calculated from the MD simulation for the elastic quantities, as is also exemplified in Fig. 2. This rules out any explanation for such phenomenon given in terms of different temperature dependences of the three partials (i.e., the increase in width at low temperature would then arise from inhomogeneous broadening). Notice however that the quantities experiencing a more marked dependence with temperature are those regarding the elastic component, as data given in Fig. 1 regarding $Q_{p}, \Delta_{Q}$ and $I_{Q}$ clearly exemplify. In this respect, it is worth noting the apparently anomalous trend followed by data regarding $\Delta_{Q}$, if considered in terms of some correlation length (i.e., in the sense of that given by a Scherrer equation) $R_{c}$, which would increase with tempera- 

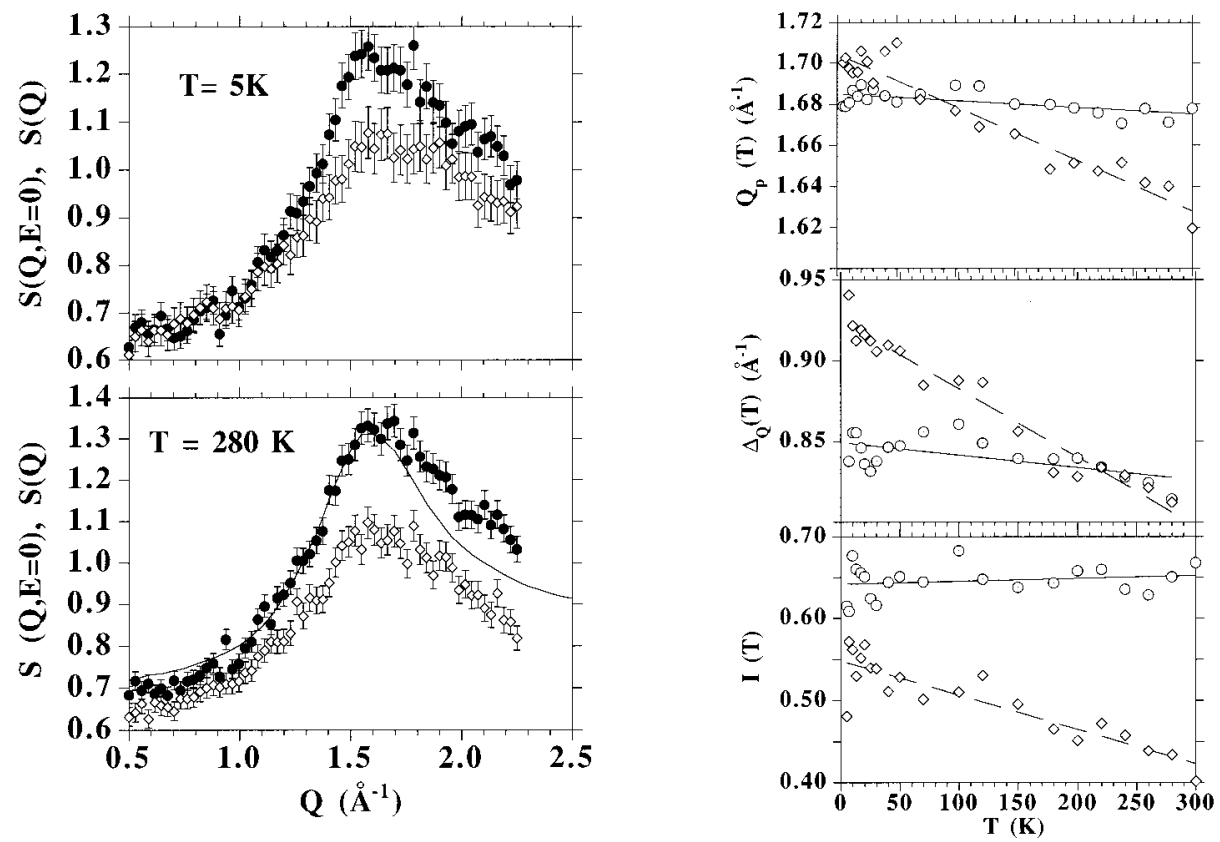

FIG. 1. The left side of the figure shows the fully corrected experimental data for $S(Q)$ (circles with a dot) and $S(Q, E=0)$ (lozenges) for temperatures of $5 \mathrm{~K}$ (top) and $280 \mathrm{~K}$ (bottom). The line drawn through the experimental data at $280 \mathrm{~K}$ represents diffraction data by Jonhson et al. (Ref. 17) using total scattering (time-of-flight) techniques. The right side shows the temperature dependence of quantities derived from fits to the experimental structure factors. Upper: dependence of $Q_{p}=2 \pi / R$, circles with a dot show data for $S$ ( $Q$ ) and lozenges those for the elastic structure factor. The lines drawn through the points represent an approximate linear temperature dependence used to describe the data. Middle: that for the peak width $\Delta_{Q}=2 \pi / R_{c}$, same symbols than before used. Lower: the temperature dependence of the integrated peak intensity.
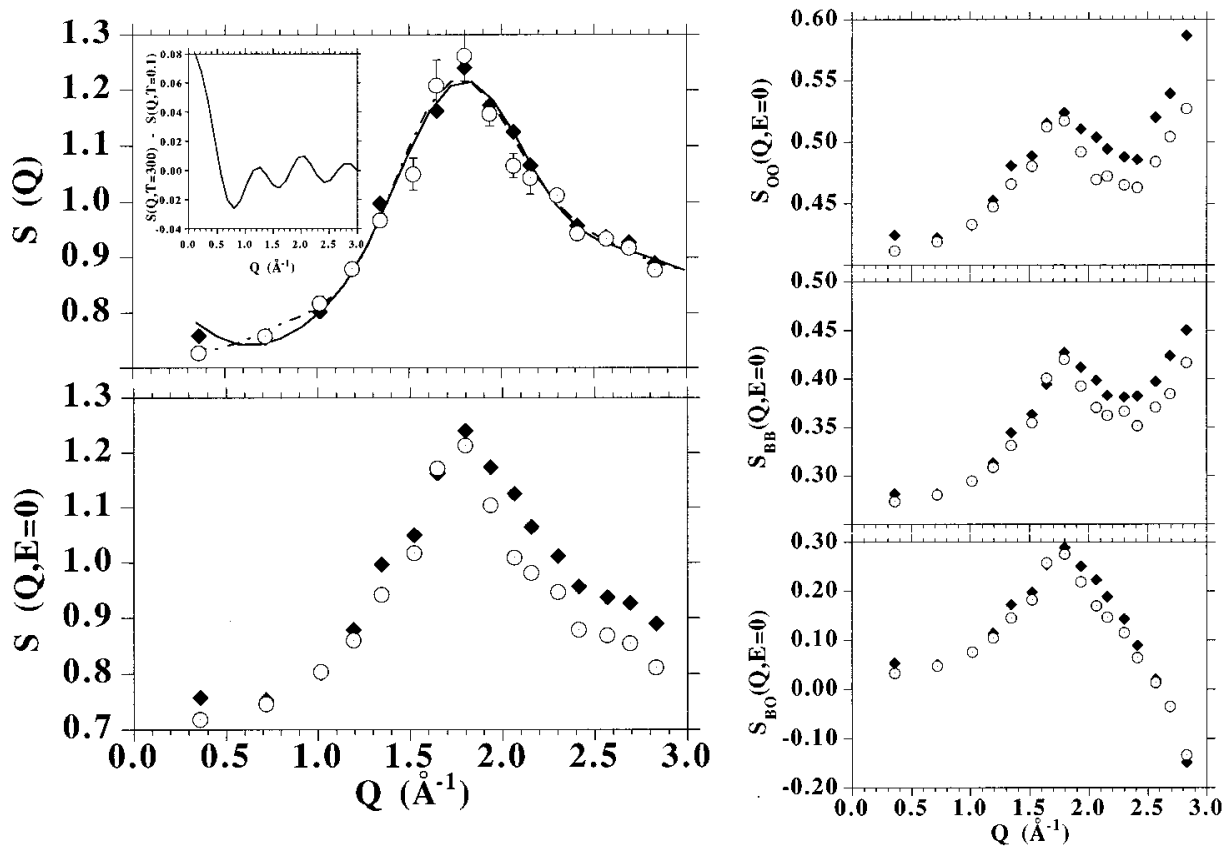

FIG. 2. The left side shows the structure factors as calculated by MD simulations for $T=0.1 \mathrm{~K}$ (filled lozenges) and $T=300 \mathrm{~K}$ (circles with a dot). Notice that the $S(Q)$ static quantities here plotted correspond to those derived by integration over frequency of the $S(Q, E)$ dynamic structure factors, while the broken line $(T=0.1 \mathrm{~K})$ and the full line $(T=300 \mathrm{~K})$ were calculated from the static pair-correlation functions (see text). The right-hand side of the graph depicts the $S_{\alpha \beta}(Q, E)$ partial dynamic structure factors as calculated from MD results for the static and elastic quantities at the two temperatures given above. The inset within the upper left frame depicts the intensity difference between structure factors for 300 and $0.1 \mathrm{~K}$. 

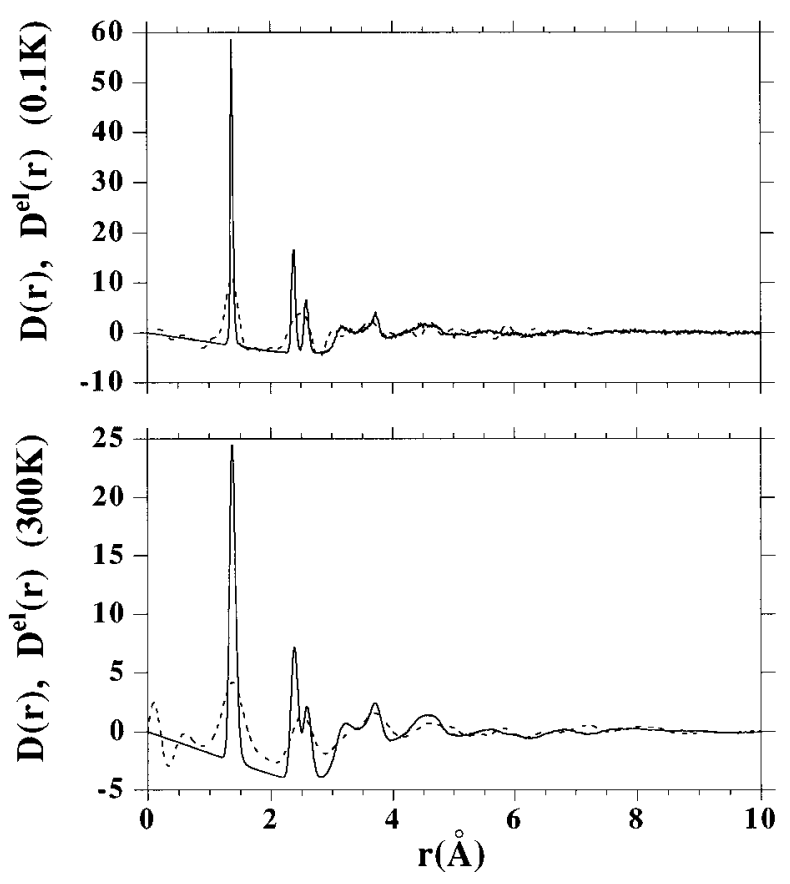

FIG. 3. $D(r)$ static and $D^{\mathrm{el}}(r)$ pair-correlation functions for $T=0.1 \mathrm{~K}$ (upper frame) and $T=300 \mathrm{~K}$ (lower frame) as calculated from the molecular-dynamics trajectories. The static quantities are depicted by solid lines and the elastic by dash-dotted lines.

ture by some $1.1 \times 10^{-3} \mathrm{~K}^{-1}$ for the elastic (and one order of magnitude below for the static) quantity.

In search of a real-space correlate of the changes appearing in the calculated structure factors, the $D(r)$ static paircorrelation functions [i.e., the Fourier pair of $S(Q)-1$ ] and that $D^{\mathrm{el}}(r)$ corresponding to the elastic $S(Q, E=0)$, function were calculated. These are shown in Fig. 3 which depicts the quantities corresponding to the static structure factors for $T=0.1$ and $300 \mathrm{~K}$. From there it can be observed that as may seem obvious, the ordering at distances below some $7 \AA$ is enhanced in the low-temperature calculation as attested by the sharper and more intense oscillations in $D^{\mathrm{el}}(r)$. In fact, from analysis of the first three well-resolved peaks corresponding to B-O $\left(r_{1}\right)$, O-O $\left(r_{2}\right)$, and B-B $\left(r_{3}\right)$ contacts, the following parameters corresponding to equilibrium positions $r_{i}$ and widths $\Delta r_{i}^{2}$ are derived $r_{i}\left(\Delta r_{i}\right)_{i=1,3}=1.376 \AA$ $\left(0.47 \times 10^{3} \AA^{2}\right), \quad 2.384 \AA \quad\left(1.08 \times 10^{3} \AA^{2}\right), \quad 2.591 \AA$ $\left(1.54 \times 10^{3} \AA^{2}\right)$, for $T=0.1 \mathrm{~K}$ and $1.379 \AA\left(2.55 \times 10^{3} \AA^{2}\right)$, $2.392 \AA\left(3.89 \times 10^{3} \AA^{2}\right)$, and $2.602 \AA\left(4.60 \times 10^{3} \AA^{2}\right)$ for $T=300 \mathrm{~K}$, respectively. Such figures for the peak widths are about $0.7 r_{1}$ and $0.5 r_{2}$ times smaller than those given by Misawa ${ }^{10}$ from analysis of experimental distributions derived from Fourier inversion of neutron data truncated at some $32-34 \AA^{1}$ and constitute a vivid illustration of the difficulty which entails the measurement of the natural width of shortrange peaks in the glass distance distribution using the means nowadays available. An estimate of the relative importance of static versus thermal fluctuations can be made by ratioing the width data given above since the width of the simulation results for $T=0.1 \mathrm{~K}$ should be fully ascribed to static disorder. In counterposition, the structure at larger distances, that is, correlations involving atoms located at positions beyond those characteristic of next-nearest neighbors, is hardly af- fected, and in fact, within some ranges of distances the function for $T=300 \mathrm{~K}$ appears somewhat more structured. This is illustrated by the lower intensity of oscillations above some 8 $\AA$ as seen in the referred figure, which also evidences that such oscillations in the $0.1 \mathrm{~K}$ function are clearly phase shifted which respect to those for $T=300 \mathrm{~K}$.

The origin of such counterintuitive result may be ascribed to the significant changes in the dynamics brought forward by the anharmonic parts of the interparticle potential, which lead to a significant volumic contraction below some $3 \mathrm{~K}$ as well as to a strong decrease of the Grüneisen parameter, which plummets from some $\gamma_{g} \approx 1$ at $T=20 \mathrm{~K}$ down to -0.3 at $T=2 \mathrm{~K}^{12}$ More specifically, and as discussed in some detail in our previous communication, ${ }^{16}$ lowering the temperature down to a few kelvins leads to a redistribution of frequencies in the $Z(E)$ generalized frequency spectrum (i.e., vibrational density of states), where the spectral power is shifted towards low frequencies. The net effect of the decrease in temperature is to shift the higher lobe of $Z(E)$ (above some $120 \mathrm{meV}$ ) towards frequencies below $25 \mathrm{meV}$ as shown in Ref. 16. Results arising from the analysis of the dynamics in terms of instantaneous normal mode, shows that this shift must be ascribed to pure anharmonic effects, since the normal-mode distributions regarding stable (real frequency vibrations), and unstable modes (modes with imaginary frequencies) showed a far weaker temperature dependence. It is worth noticing that this remarkable effect on frequency redistribution with temperature has also been detected in recent inelastic neutron-scattering work. ${ }^{18}$

To delve into the origin of such a change in structure with temperature as well as to help to clarify the origin of the FSDP, we have subtracted from the calculated total $g(r)$ static pair-correlation function the intensity $f(r)$ arising from a stoichiometric $\mathrm{B}_{2} \mathrm{O}_{3}$ unit, so that the resultant $g_{\text {inter }}(r)=g(r) f(r)$ comprises correlations between atoms pertaining to different $\mathrm{B}_{2} \mathrm{O}_{3}$ units. In doing so, a parallelism with the usual procedure employed for the separation of inter- and intramolecular correlations in one of the conventional approaches to the analysis of the diffraction patterns of molecular materials ${ }^{19}$ is followed. This is facilitated by the fact that the structure of such units is relatively well defined since, as illustrated in Fig. 2 of Ref. 20 even the relatively large $\mathrm{BO}$ nonbonded distances within that unit are grouped within rather narrow ranges. The advantage of pursuing such a route stems from the fact that once the short-range structure has been removed what remains,

$$
\begin{aligned}
D(Q) & =1+4 \pi \rho \int_{0}^{\infty}\left[g_{\text {inter }}(r)-1\right] j_{0}(Q r) r^{2} d r \\
& =S(Q)-f(Q)
\end{aligned}
$$

represents a structure factor $D(Q)$ for 'intermolecular', contacts which can be rightly interpreted in terms of orientational and center of mass correlations between moleculelike entities and therefore the information regarding order at intermediate distances can be discussed on a more quantitative ground. To proceed, a set of Gaussians corresponding to $\mathrm{B}-\mathrm{O}, \mathrm{B}-\mathrm{B}$, and $\mathrm{O}-\mathrm{O}$ contacts within such a chemical unit were fitted to the calculated $g_{\alpha \beta}(r)$ partial pair correlations, leaving their positions and widths as adjustable parameters, 

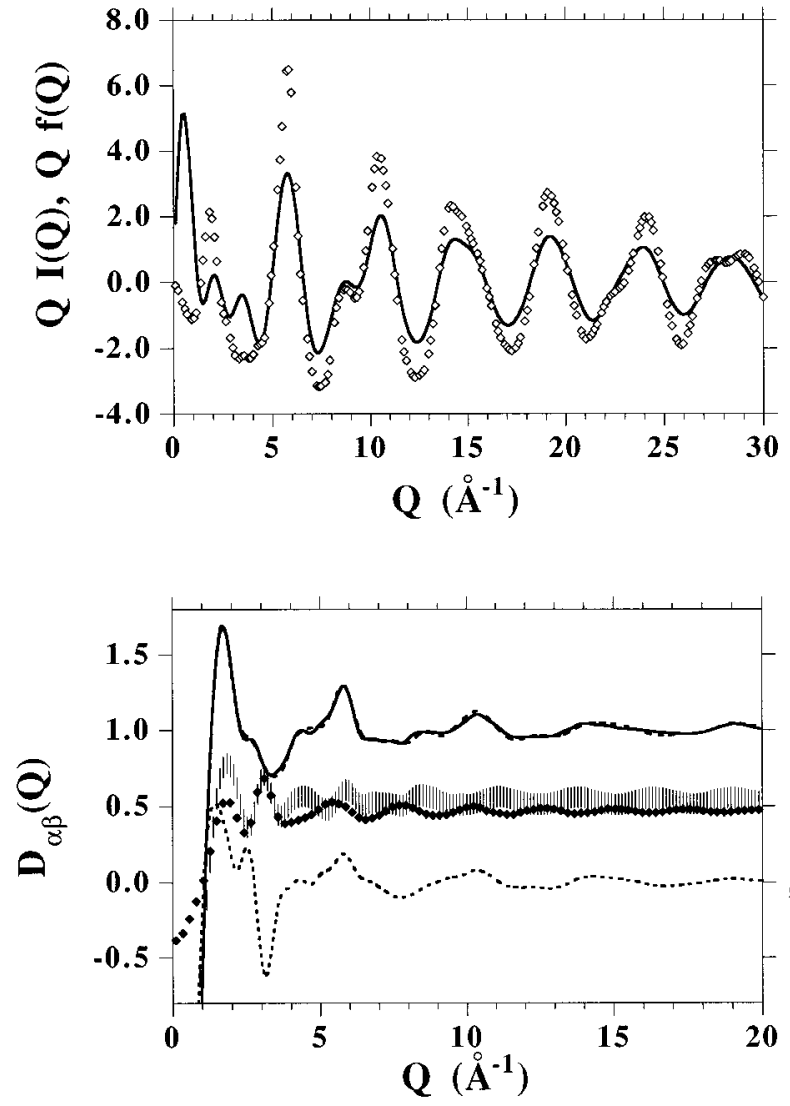

FIG. 4. The upper frame depicts a comparison between the calculated $Q I(Q)$ reduced interference function (lozenges) with the $Q f(Q)$, structure factor corresponding to $\mathrm{a}_{2} \mathrm{O}_{3}$ stoichiometric (chemical) unit (see text). The lower frame shows the "intermolecular' structure factors (i.e., those derived after subtraction of the shortrange, $f(Q)$ structure factor. The solid line shows the total $D(Q)$ function, and the different partial structure factors corresponding to this are given by vertical bars $\left[S_{\mathrm{OO}}(Q)\right]$, filled lozenges $\left[S_{\mathrm{BB}}(Q)\right]$ and a dotted line $\left[S_{\mathrm{BO}}(Q)\right]$. The dashed line close to $D(Q)$ displays the quantity calculated from data pertaining to the simulation at $0.1 \mathrm{~K}$.

but having their relative intensities set to fixed ratios. The result of such an exercise is shown in Fig. 4 where the $Q I(Q)=Q[S(Q)-1]$ reduced interference function and that $Q f(Q)$ corresponding to the chemical unit, that is the one calculated by Fourier inversion of $f(r)$, are compared. As shown there, both functions oscillate inphase for distances above some $5 \AA^{-1}$, whereas a rather distinct behavior is clearly seen below such wave numbers, especially below some $3 \AA^{-1}$. Comparison of the intensity about $Q$ values characteristic of the FSDP $\left(1-2 \AA^{-1}\right)$ shows that a small but non-negligeable contribution to the intensity of such a peak arises from short-range structures.

Once the contributions comprised in $f(Q)$ are subtracted, it is seen that, as evidenced in the lower frame of Fig. 4, what remains of the FSDP constitutes the most intense peak in the “intermolecular" $D(Q)$ function. Also, the intensity of such a peak arises from oscillations from the partial $D_{\alpha \beta}(Q)$ functions which show peaks in the $1.5-2 \AA^{-1}$ range of momentum transfers. Above some $5 \AA^{-1}$ most of the features identifiable as distinct peaks within $D(Q)$ mostly arise from the
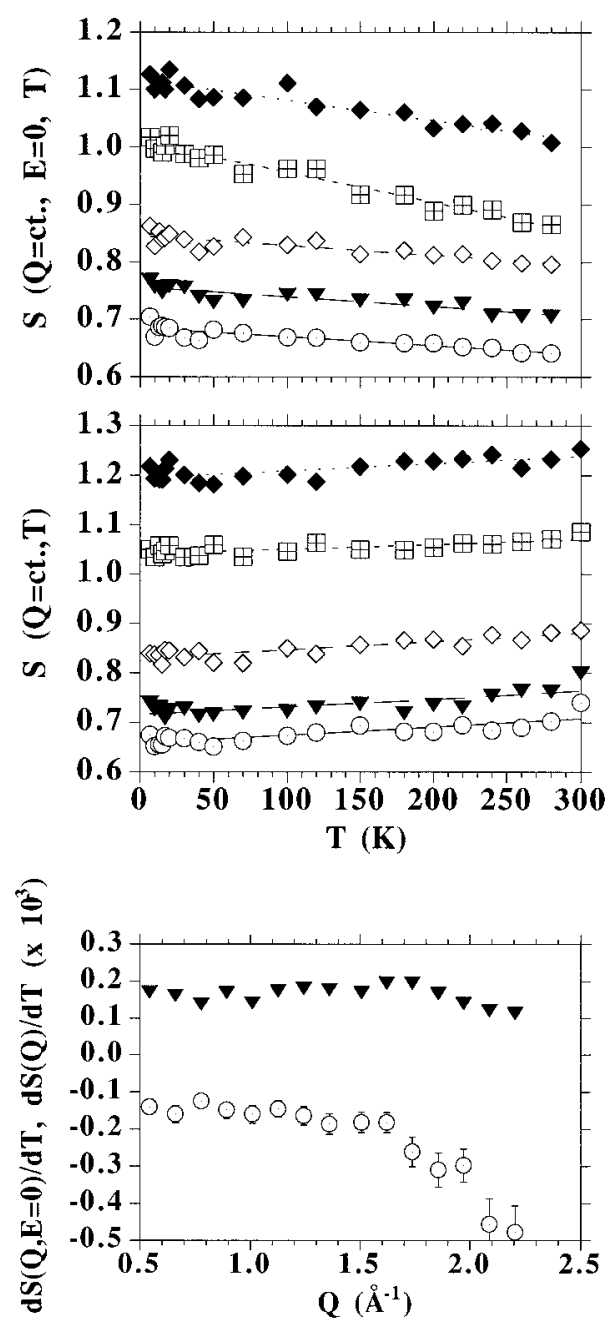

FIG. 5. Temperature dependence of the intensities in the experimental elastic (up) and static (middle) structure factors for a selected set of wave numbers. The straight lines represent linear fits used to extrapolate the data to $T=0 \mathrm{~K}$ as well as to get some estimate of the temperature derivatives of both structure factors. The different symbols represent data for $Q=0.55 \AA^{-1}$ (circles with a dot), $0.89 \AA^{-1}$ (filled inverted triangles), $1.13 \AA^{-1}$ (lozenges), $2.21 \AA^{-1}$ (squares with a cross) and $1.86 \AA^{-1}$ (filled lozenges) The lower frame shows the estimates for the dependence with the wave number of the temperature derivatives of both structure factors. The temperature derivatives of $S(Q)$ are shown by filled inverted triangles and those for $S(Q, E=0)$ by circles with a dot.

$D_{\mathrm{BO}}(Q)$ partial function since those arising from $\mathrm{BB}$ and $\mathrm{OO}$ are nearly perfectly out-of-phase. In the same figure a comparison of the calculated $D(Q)$ at 0.1 and $300 \mathrm{~K}$ is shown. The small effect of such a large change in temperature on the resulting $D(Q)$ functions becomes basically identical to that shown by $S(Q)$ as mentioned in above paragraphs and illustrated in the inset of Fig. 2.

\section{B. Debye-Waller factors}

The data shown in the Fig. 5 serve to quantify the dependence with wave numbers of the change in intensity brought forward by increasing the temperature from $5 \mathrm{~K}$ upwards. It becomes clear by visual inspection of such a graph that the 
marked decrease in the intensity of the elastic structure factor above $Q_{p}$ is not entirely compensated by the increase in the intensity of the static one. Such effect is clearly exemplified by estimates for the temperature derivatives of both structure factors shown in the bottom frame of Fig. 5. This process is basically originated by a transfer of intensity from the elastic to inelastic components of the spectra, and to quantify such a fact the following functions:

$$
f(Q, T)=\frac{S(Q, E=0)(T)}{S(Q, E=0)(T=5 \mathrm{~K})}\left[\frac{S(Q)(T)}{S(Q)(T=5 \mathrm{~K})}\right]^{-1},
$$

which are an approximation to the DebyeWaller factor, were considered. Notice that in order to minimize the errors arising from ratioing two quantities which are not easy to normalize to an absolute scale, both $S(Q, E=0)$ and $S(Q)$ are scaled to the lowest temperature datasets. An immediate consequence of such a procedure would be to remove a rather substantial part of the oscillations arising from both $S(Q, E=0)$ and $S(Q)$, and therefore the $f(Q, T)$ functions so obtained are expected to be closer to the incoherent Lamb-Mössbauer factors than to the coherent quantities (Debye-Waller terms in strict sense, although we will keep such nomenclature to be consistent with most of the available literature). Some results depicting the wave-vector and temperature dependences of $f(Q, T)$ are shown in Fig. 6. Although the statistical noise in $f(Q, T)$ is large, some general remarks concerning the measured data can be made. As expected, the data shown in the figure as $\ln f(Q, T)$ versus $Q^{2}$ do not show any clear oscillatory behavior as found for other covalent glasses such as $g$-Se, ${ }^{4}$ and, in fact, on approximate grounds, the wave-vector dependence of such quantities can be accounted for in terms of the incoherent approximation [i.e., $\ln f(Q, T) \propto Q^{2}$ ]. Such a behavior is followed by data at low temperatures (i.e., below some $150 \mathrm{~K}$ ), as judged by the randomness of the distribution of residuals. As shown in the graph, at temperatures of $200 \mathrm{~K}$ (that is some 0.37 $T_{g}$ ) and above, most of data lay below the fitted line and in fact, the intercept at $Q \rightarrow 0$ is significantly smaller than that corresponding to $f(Q, T)=1$.

To assess the reliability of the measured data and the ensuing approximations followed for their analysis, the slopes derived from fits described in the above paragraph are compared with quantities calculated using a simple Debye approximation $^{21}$

$$
2 W_{D}(Q)=\frac{3 \hbar Q^{2}}{M_{a v} \omega_{D}^{3}} \int_{0}^{\omega_{D}} \omega\left(\frac{1}{\exp \left(\hbar \omega / k_{B} T\right)-1}+\frac{1}{2}\right) d \omega .
$$

Setting the $\omega_{D}$ Debye frequency to the value derived from calorimetric means, ${ }^{12} \omega_{D}=k_{B} \theta_{D} / \hbar=5.397 \mathrm{THz}$ and that for the atomic mass to an average value taken over the isotope composition $M_{\mathrm{av}} \approx 14 \mathrm{amu}$, the above equation provides values for the exponent entering the Debye-Waller term and therefore for the average atomic mean-squared displacements $\left\langle u^{2}(T)\right\rangle$, close to those derived from experimental means, as Fig. 6 shows. The agreement is particularly good within the range $125-280 \mathrm{~K}$, which comprises $\theta_{D}$. However, the most systematic deviation occurs below $50 \mathrm{~K}$, a temperature region where anharmonic effects as reflected by the macroscopic Grüneisen function become significantly large. ${ }^{12}$ Sur-
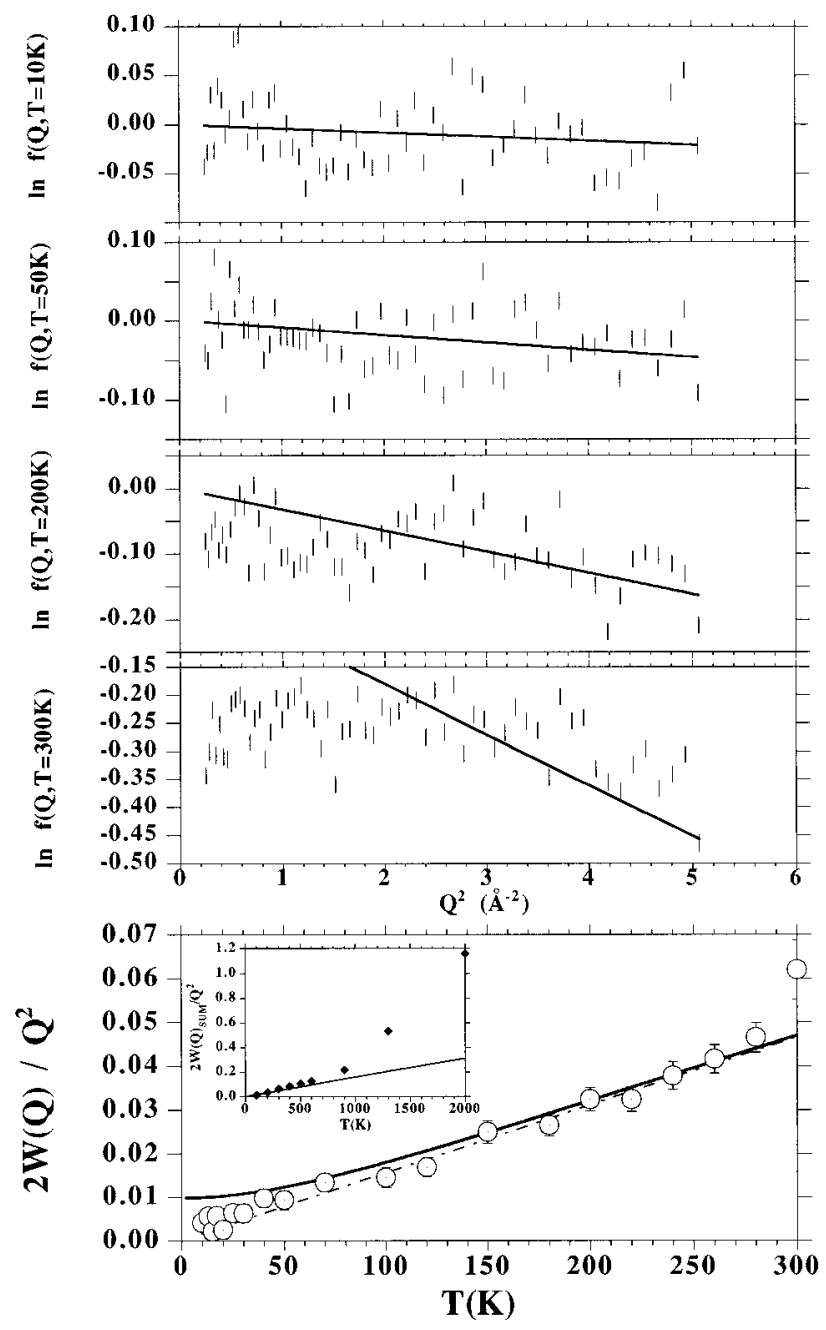

FIG. 6. The upper four frames show the wave-vector dependence of the experimental $\ln f(Q, T)$ for several temperatures (vertical bars). The straight lines represent fits to a quadratic law $2 W(Q)=A Q^{2}$ (see text). The bottom frame shows the resulting coefficients (circles with a dot), which are compared with a Debye approximation [Eq. (3), full line], as well as with an extrapolation of a hightemperature approximation (dash-dotted line, see text). The inset in this latter graph depicts a comparison of the average atomic displacement as calculated for temperatures at both sides of the glass transition (filled lozenges) and the hightemperature approximation (solid) for the vibrational part as calculated from the Debye model.

prisingly, a simple high-temperature estimate for these quantities, that is $W_{D}(Q)=3 \hbar^{2} T / M_{a v} k_{B} \theta_{D}^{2}$ calculated using the constants given above follows the data better than the complete expression given by Eq. (3) (see Fig. 6).

However, for temperatures above some $150 \mathrm{~K}$ a significant departure of $f(Q, T)$ from such simple quadratic behavior in $Q$ is evidenced by the systematic deviation from the linear dependence of $\ln f(Q, T)$ versus $Q^{2}$ as well as by the decrease of elastic intensity with increasing temperature which becomes specially noticeable at low wave vectors. This is a manifestation of the fact that at wave vectors well below $Q_{p}$ the decrease with temperature of $S(Q, E=0)$ $\left(\approx 1.4 \times 10^{3} \mathrm{~K}^{-1}\right)$ is concomitant with a somewhat larger increase in $S(Q)\left(\approx 1.4 \times 10^{3} \mathrm{~K}^{-1}\right)$. The strong falloff which 
is readily visible at wave vectors somewhat above $Q_{p}$ is, on the other hand, originated by a similar feature in the elastic structure factor which is absent in the normalized $S(Q)$.

To ascertain the microscopic origin of such decrease in elastic intensity at high temperatures, recourse was made to the MD simulation in order to check (a) whether higherorder terms in the phonon expansion of the Debye-Waller term (i.e., apart from the leading harmonic $Q^{2}\left\langle u^{2}\right\rangle / 3$ one) could be of importance at temperatures close or above $\theta_{D}$, and (b) to explore the possibility of the existence of lowfrequency motions of relaxational or other types which may give rise to some quasielastic broadening. The former were evaluated by means of the computation of cubic and quartic terms in $Q$, that is ${ }^{22}$

$$
\begin{aligned}
W(Q)= & \frac{1}{6}\left(Q^{2}\left\langle u^{2}\right\rangle-i\left\langle(\mathbf{Q} \cdot \mathbf{u})^{3}\right\rangle-\frac{1}{4}\left[\left\langle(\mathbf{Q} \cdot \mathbf{u})^{4}\right\rangle\right.\right. \\
& \left.\left.-3\left\langle(\mathbf{Q} \cdot \mathbf{u})^{2}\right\rangle^{2}\right]\right),
\end{aligned}
$$

from results pertaining to the atomic Cartesian displacements as calculated from the normalmode decomposition described in Ref. 16. Notice that the bold-faced vector products in the formula given above stand for orientational averages for which explicit (but lengthy) expressions were derived. The relative importance of the different terms versus $Q$ for the two extreme temperatures considered in Ref. 16 is depicted in Fig. 7. The cubic term although being finite (only vanishes in cases where every atom is a center of inversion symmetry) becomes three orders of magnitude smaller than the quartic one and was therefore discarded. Inspection of Fig. 7 thus shows that a substantial anharmonic contribution to $W(Q)$ can be expected at momentum transfers beyond $\approx 2 \AA^{-1}$ at $T=300 \mathrm{~K}$, being such an effect easy to detect by a deviation from linearity in plots of $\ln f(Q)$ versus $Q^{2}$. On the other hand, all other effects arising from thermal expansion will contribute to the total $W(Q)$ by an amount $W^{\mathrm{qh}}(Q)$ which can easily be estimated within a quasiharmonic approximation $^{22}$

$$
W(Q)=W^{h}(Q)\left(1+2 \gamma_{G} \chi T\right)=W^{h}(Q)+W^{\mathrm{qh}}(Q),
$$

where $W^{h}(Q)$ refers to the quantity calculable on harmonic grounds, $\gamma_{G}$ is the Grüneisen constant for a given temperature $T$, and $\chi$ is the cubic expansion coefficient. An evaluation of the relative importance of this latter contribution with respect to those given by Eq. (4) can be readily made from thermal-expansion and specific-heat data due to White et al. ${ }^{12}$ Taking as an example the higher-temperature datum $(283 \mathrm{~K})$ measured by those authors one finds an anharmonic contribution as given by $2 \gamma_{G} \chi T_{283 \mathrm{~K}}=$ of some $7.2 \times 10^{-4}$, a number which can safely be discarded when compared with $W^{h}(Q)$, although such a term has to be taken into account if data close to the glass-transition region (above some $500 \mathrm{~K}$ ) are considered. To provide an independent test of the deviation from ideality of $W(Q)$, the departure of the atomic displacements from Gaussian behavior was estimated from the calculated moments of the van Hove $G_{s}(r, t)$, selfcorrelation function. Such moments,
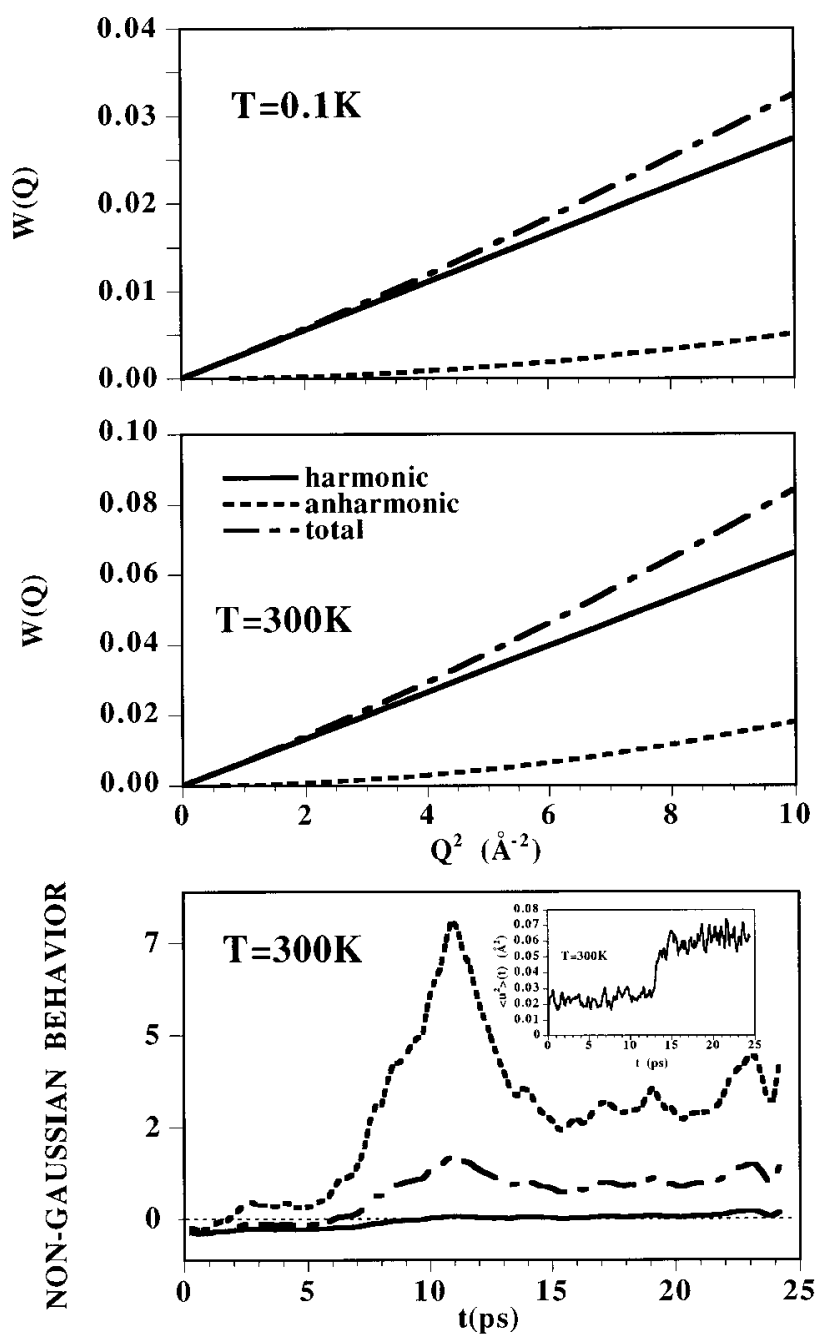

FIG. 7. The upper and middle frames show a comparison between harmonic (i.e., quadratic in $Q$ ) and anharmonic (quartic $Q$ dependence) contributions to the $W(Q)$ exponent entering the Debye-Waller term as calculated from Eq. (4). The lower frame shows values of the moments $\alpha_{n}(t)$ as calculated from Eq. (6) giving estimates of non-Gaussian behavior of the time-dependent pair-correlation function. Curves shown correspond to $n=2$ (solid), $n=3$ (dash-dot), and $n=4$ dots. The inset shows the time dependence of the mean-squared atomic displacement calculated in the same simulation run.

$$
\begin{gathered}
\alpha_{n}(t)=\left(\left\langle r^{2 n}\right\rangle / C_{n}\left\langle r^{n}\right\rangle^{n}\right)-1 ; \\
C_{n}=(2 n+1) ! ! / 3^{n} ;\left\langle r^{2 n}\right\rangle=\int d r r^{2 n} G_{s}(r, t)
\end{gathered}
$$

will only vanish for Gaussian behavior. The curves showing the dependence of $\alpha_{2}, \alpha_{3}$ and $\alpha_{4}$ are also drawn in Fig. 7. The most remarkable facts about such curves regard their negative values at short times which is indicative of a decay faster than Gaussian, the presence of strong peak at some 11 ps which seems to be related with the onset of a strong jump of the average displacement shown in the inset, and finally their approach to some finite value at long times which is again indicative of a departure from Gaussian behavior, this time corresponding to a decay slower than Gaussian. 

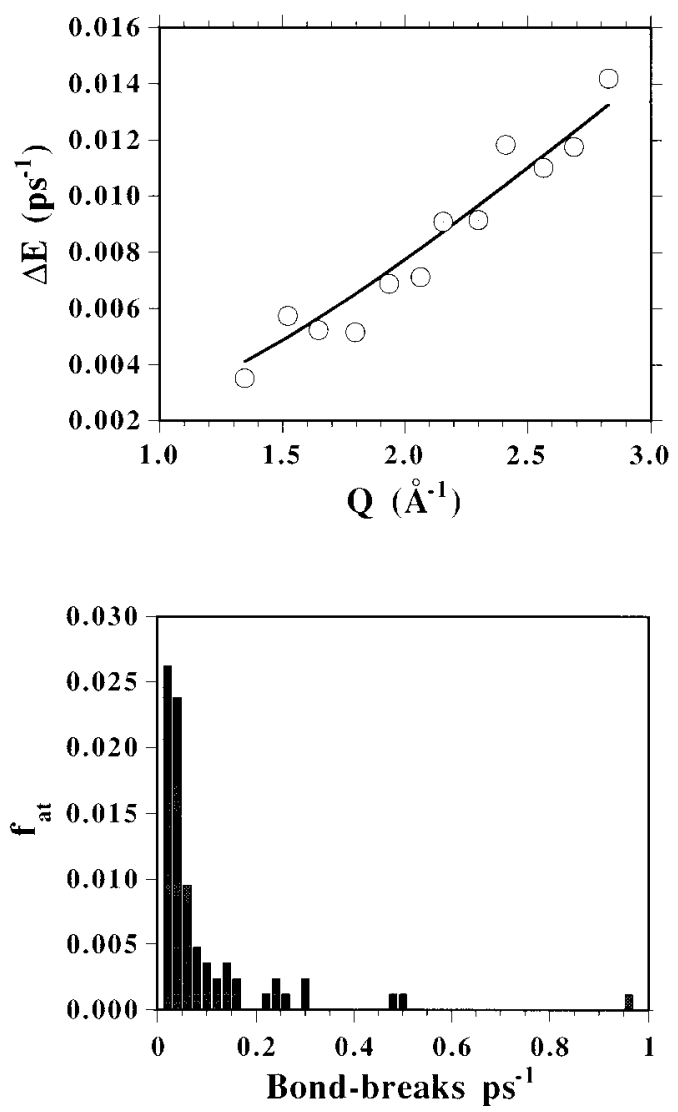

FIG. 8. The upper frame shows the wavevector dependence of the quasielastic broadening for $T=300 \mathrm{~K}$ (circles with a dot). The solid line shows a parametric approximation to the data using Eq. (8) (see text). Lower frame shows an histogram displaying the number of broken bonds per picosecond. The quantity is normalized to the total number of atoms in the simulation box (420).

On the other hand, a visual comparison of the calculated $S(Q, E)$ functions for both temperatures for energy transfers of a few meV about the elastic line immediately shows the presence of a spectral component of either zero or very low frequency which cannot be resolved within the time scales of the simulation. The presence of such an apparent broadening of the elastic peak was unexpected since at temperatures well below the glass transition $\left(0.37 T_{g}\right.$ and below $)$ the glassy dynamics is customarily portrayed as mostly driven by vibrations, ${ }^{23}$ and therefore such an aspect has not been previously considered in detail, although the presence of quasielastic broadening is evident in many glasses above some $0.6-0.8 T_{g}$. The wave-vector dependence of such a broadening could only be resolved for $Q \geqslant Q_{p}$ and is shown in Fig. 8. Although a definite conclusion regarding the $Q$ dependence of this quantity could not be reached due to the relatively poor statistics, the data above $Q_{p}$ seem to be compatible with predictions based upon simple models of particle motion in an anharmonic potential, ${ }^{5}$ where such a broadening of the elastic line is shown to vary with $Q^{2}$. On the other hand, the availability of the MD trajectories should, in principle, enable one to investigate the origin of such a broadening on a microscopic basis. Since such a quasielastic component cannot arise from any kind of harmonic motion, including those corresponding to unstable (i.e., imaginary frequency) modes described in some detail in our previous work, ${ }^{16}$ we set out to quantify other kind of motions which, due to the low frequencies involved, may give rise to large-amplitude motions. In fact, the wave-vector dependence of the broadening could be interpreted as an indication of the onset of particle diffusion at such low temperatures, ${ }^{24}$ a phenomenon which has been predicted to take place in disordered media and which has received considerable attention in recent times. If this were the case, such motions which would conceivably involve particle jumps over short distances, should be preceded by the breaking of some bonds, leaving the particle free to move until trapped and forming a bond again. To proceed quantifying the relative importance of such mechanism we carried out a statistical analysis of a network structure formed by 420 atoms following the bondbreaking and forming processes for 65 ps. To do this, a simple geometrical criterium to decide whether a bond was intact or not was adopted. According to this, a B-O bond was considered as broken when the distance between both nuclei exceeded a distance of $1.7 \AA$, a value which was taken upon calculating the $D_{\mathrm{BO}}(r)$ to get the correct number of first neighbors. The result of such a search is shown in Fig. 8. From there it is seen that a small but non-negligeable fraction of atoms (about $10 \%$ ) are involved in bond-breaking processes which take place with mean frequencies of $8 \times 10^{4}$ bond breaks per picosecond The total mean-squared displacement per particle should then comprise a contribution from vibrations, whether harmonic or not, and another from spatial excursions performed after breaking some bonds. Lets retain the nomenclature of Bhattacharya et al. ${ }^{5}$ and name $\left\langle u_{\mathrm{vib}}^{2}\right\rangle$ to the former and $\left\langle u_{\mathrm{qe}}^{2}\right\rangle$ to the latter, the subscript meaning that this quantity serves to specify the transfer of intensity from the elastic line to the quasielastic part of the spectra, and is defined as the difference ${ }^{5}$

$$
\left\langle u_{\mathrm{qe}}^{2}\right\rangle(T)=\left\langle u_{\text {sum }}^{2}\right\rangle(T)-\left\langle u_{\mathrm{vib}}^{2}\right\rangle(T),
$$

where $\left\langle u_{\text {sum }}^{2}\right\rangle$ stands for the total atomic displacement. Determination of this latter quantity is in our case easily achieved from analysis of the calculated trajectories. In fact, results regarding this quantity as calculated for temperatures between 100-2000 $\mathrm{K}$ are shown in the inset of the bottom frame of Fig. 6. There, it can be seen that a clear change in slope of $\left\langle u_{\text {sum }}^{2}\right\rangle(T)$ is present somewhat above $500 \mathrm{~K}$, marking the onset of the glass transition. From the difference between such quantities and those given for the vibrational contribution by the Debye model, estimates for $\left\langle u_{\mathrm{qe}}^{2}\right\rangle(T)$ are derived. These show that the onset for quasielastic scattering may be located about some $200 \mathrm{~K}$ where a value of $\left\langle u_{\text {qe }}^{2}\right\rangle_{200 \mathrm{~K}}=0.0037 \AA^{2}$, and $\left\langle u_{\mathrm{qe}}^{2}\right\rangle_{300 \mathrm{~K}}=0.0156 \AA^{2}$, while a value as large as $0.03 \AA^{2}$ is found at $500 \mathrm{~K}$ (close but below $T_{g}$ ). This being the case, the ratio $\left\langle u_{\mathrm{qe}}^{2}\right\rangle /\left\langle u_{\text {sum }}^{2}\right\rangle$ gives, as stated in Ref. 5, a measure of the fraction of the inelastic intensity of a quasielastic nature and gives some $11 \%$ at 200 $\mathrm{K}$ and up to $25 \%$ at $300 \mathrm{~K}$, two figures able to explain the drop of $f(Q, T)$ apparent in the higher temperature sets shown in Fig. 6.

In order to relate the frequencies of bond breaking with a quasielastic width, we have followed some recipes employed previously for the analysis of the stochastic dynamics of dense (hydrogen-bonded) liquids, ${ }^{25}$ which is described as a 
composite Gaussian process where the atom is subjected to collective vibrations during a time $\tau_{0}$ while it remains bonded to other particles and executes free diffusive motion for a time $\tau_{1}$. Under the circumstances of our interest, and taking into account the simulation results quoted above, $\tau_{1} \ll \tau_{0}$, and in this particular case, the quasielastic spectrum becomes of a Lorentzian shape with a linewidth given by Ref. 25,

$$
\Delta E(Q)=\frac{1}{\tau_{0}}\left[1-\frac{\exp (-2 W(Q))}{1+Q^{2} D \tau_{0}}\right] .
$$

Since the "diffusion coefficient" $D$ may be equated to $\left\langle u_{\text {sum }}^{2}\right\rangle / 6 \tau_{0}$, the only parameter which needs to be supplied is the residence time $\tau_{0}$. An estimate for such a characteristic time may be obtained from the bond-breaking statistics described above. From there it is found from the calculated probability for a bond to remain intact $p_{B}=0.42$ that the value for the associated $\tau_{0}$ should be of about $22 \mathrm{ps}$. As an alternative, the quasielastic linewidths calculated from simulation data can be analyzed using Eq. (8), leaving $\tau_{0}$ as an adjustable parameter. As shown in Fig. 8, such an approximation suitably accounts for the wave-vector dependence of the linewidth and gives an estimate of $\tau_{0} \approx 28 \mathrm{ps}$, in tolerable agreement with the time calculated from analysis of the bond breaks.

\section{Temperature dependence of the intensities}

To consider in some detail the variation with temperature of both static and elastic functions, recourse is made to a phonon expansion of the structure factor following steps analogous to those employed in previous works. ${ }^{4}$ As customarily done, the static structure factor is written in terms of the phonon expansion as

$$
\begin{aligned}
S(Q)= & \exp (-2 W(Q))\left[S_{R}(Q)+2 W(Q) S^{1}(Q)\right. \\
& \left.+\frac{[2 W(Q)]^{2}}{2 !} S^{2}(Q)+\cdots\right],
\end{aligned}
$$

where

$$
S_{R}(Q)=\frac{1}{N} \sum_{l, m} j_{0}\left(Q r_{l m}\right)
$$

is a "rigid" structure factor ${ }^{26}$ calculable from simulations as a sum over all $l, m$ pairs of $N$ particles separated by $r_{l m}$ distances, or by extrapolation of experimental data to zero temperature and correction for zero point motion as done for $g$-Se. ${ }^{4}$ The $S^{n}(Q)$ are the $n$-phonon structure factors, and every one of those contribute with a weight $[2 W(Q)]^{n} / n !$. Since estimates for $2 W(Q)$ for the range of temperatures of interest have been discussed in the above paragraphs, some bounds for the number of terms to be kept can be given. In fact, consideration of the values shown in Fig. 6 for the higher temperatures shows that the ratios $S^{n}(Q) / S^{1}(Q)$ for $n=2,3,4$ are of $2.3 \times 10^{2}, 3.7 \times 10^{4}$ and $4.3 \times 10^{6}$, so that terms up to $n=4$ should, in principle, be included. Since, as discussed above, a Debye approximation seems to count on semiquantitative terms for the observed variation with tem-

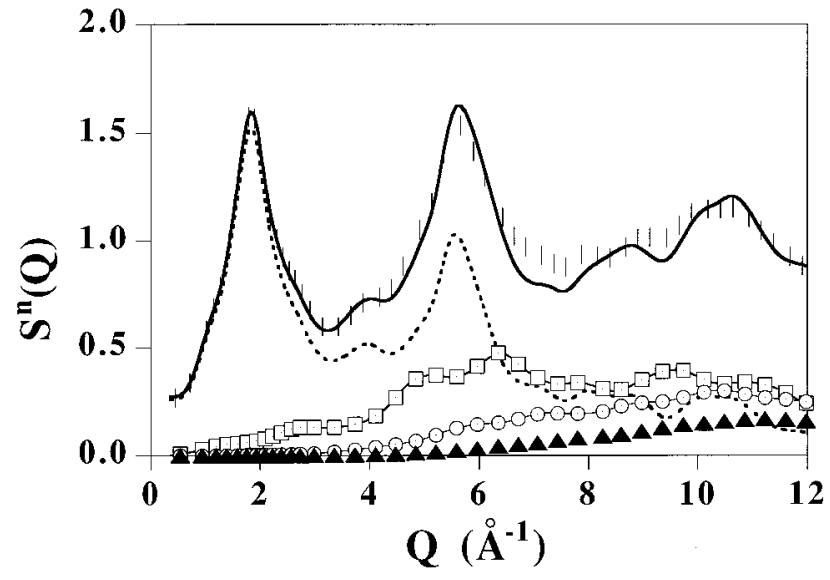

FIG. 9. Different terms entering the phonon expansion of the structure factor for $T=300 \mathrm{~K}$. The total $S(Q)$ structure factor as calculated as zeroth frequency moment of $S(Q, E)$ is shown by the thick solid line, and that arising from the phonon expansion Eq. (9) is depicted by vertical bars. The dotted line shows the zeroth phonon (elastic) structure factor, the squares with a dot the one-phonon component, circles with a dot show $S^{2}(Q)$ and filled triangles stand for $S^{3}(Q)$.

perature of $f(Q, T)$, it was taken as a starting point of the analysis. Within such a continuum model, evaluation of the $n$-phonon structure factors proceeds according to ${ }^{26,27}$

$$
S^{n}(Q)=\frac{\int_{0}^{q_{D}} q(2 n(q)+1) d q(1 / 4 \pi) \int S^{n-1}(Q+q) d \Omega}{\int_{0}^{q_{D}} q(2 n(q)+1) d q}
$$

where $q, n(q)$ stand for the wave number and population factor $n(q)=\left[\exp \left(\hbar \omega / k_{B} T\right)-1\right]^{-1}$ of phonons of frequency $\omega=v_{l} q$ within a continuum which is modeled as a sphere of radius given by the Debye wave vector $q_{D}=\omega_{D} / v_{l}=0.978$ $\AA$, where $v_{l}$ stands for the longitudinal sound velocity. A graph displaying the relative importance of terms up to $S^{3}(Q)$ in the expansion given above is shown in Fig. 9. It is there seen that such an expansion gives adequate account of the static $S(Q)$, at least for temperatures as high as $300 \mathrm{~K}$. As expected, the inelastic contributions to the FSDP are rather small and basically structureless, whereas those for the second peak in $S(Q)$ become far more marked. Also, notice that within the range of wave vectors of interest, keeping terms up to $n=3$ suffices. From such expansion, estimates for the temperature dependence $\partial S(Q) / \partial T$ can be easily calculated after partial differentiation of terms in Eq. (9). It can easily be shown that in common with results given in previous papers, ${ }^{4}$ the leading terms of the derivative referred above are those multiplied by $\partial \exp (-2 W(Q, T)) / \partial T$. Those involving partial derivatives $\partial S_{R}(Q) / \partial T$ are of the order of those given by the thermal-expansion coefficient, and by construction all those involving terms $S^{n}(Q)$ have to be of comparable magnitudes.

A comparison between the experimental data shown in Fig. 5 and those calculated by the use of Eq. (9) can now be pursued. In general terms, both trends are adequately accounted by the simulation results, that is the $S(Q, E=0)$ decreases continuously with temperature whereas $S(Q)$ shows 
a small increase in intensity, showing such variations of both structure factors a noticeable dependence with wave vector. In particular, data shown in Fig. 5 which show that $S(Q)$ has a temperature dependence of about $0.15 \times 10^{-3} \mathrm{~K}^{-1}$ with a mild $Q$ dependence, compare favorably with estimates calculated by the procedures described above, which yield values of $0.13 \times 10^{-3} \mathrm{~K}^{-1}$ for $Q=1.02 \AA^{-1}$ or $0.17 \times 10^{-3}$ $\mathrm{K}^{-1}$ for $Q=2.16 \AA^{-1}$. On the other hand, the temperature dependence of the elastic quantity is significantly underestimated by the simulation, and yields $0.33 \times 10^{-4} \mathrm{~K}^{-1}$ and $0.35 \times 10^{-4} \mathrm{~K}^{-1}$ for the same two $Q$ values, which are one order of magnitude smaller than experiment.

\section{CONCLUSIONS}

From decomposition of the structure factor in terms of short and medium-range correlations it has been shown that the FSDP basically represents the strongest peak of the structure factor for correlations between different $\mathrm{B}_{2} \mathrm{O}_{3}$ units, comprising positional correlations for distances above some $3 \AA$. Its temperature dependence can be understood in terms of a phonon expansion, at least on a semiquantitative basis, and most of it is shown to be governed by that arising from the Debye-Waller terms. The latter are shown to include at least for moderate temperatures, sizeable contributions arising from anharmonic processes leading to measurable deviations from simple Gaussian behavior.
One of the most relevant results of the present study regards the detection, by means of the concurrent use of neutron-scattering measurements and computer moleculardynamics experiments, of dynamical processes taking place well within the glass phase, which become detectable at temperatures as low as $\approx 0.25 T_{g}$, and cannot be regarded as arising from finite-frequency vibrations. Such a result contrasts with the idealized picture of the dynamics of glasses well below $T_{g}$ as portrayed by some theoretical attempts developed to account for the dynamics about the glass transition $^{28}$ but provides an experimental confirmation to predictions of phenomenological nature. ${ }^{5}$ Such findings, although preliminary, may in the future give some clues regarding the puzzling behavior of several properties such as sound attenuation, ${ }^{13}$ or the large depolarization observed in Raman peaks at about 100-150 K, which occur within the same temperature range where the onset of bondbreaking processes is found.

\section{ACKNOWLEDGMENTS}

Work was performed in part under D.G.I.C.Y.T (Spain) Grant No. PB92-0114-C03-01. Financial support from the Large Scale Facilities Programme of the European Union for the measurements carried at Risoe National Laboratory (Denmark) is acknowledged. J.D. wishes to thank CONICET (Argentina) and CSIC (Spain) for financial support.
${ }^{1}$ P.H. Gaskell and D.J. Wallis, Phys. Rev. Lett. 76, 66 (1996) and references therein.

${ }^{2}$ See, for instance A.P. Sokolov, E. Rössler, A. Kisliuk, and D. Quitmann, Phys. Rev. Lett. 71, 2062 (1993); P.S. Salmon, Proc. R. Soc. Lond. A 445, 351 (1994).

${ }^{3}$ J. Bletry, Philos. Mag. B 62, 462 (1990), S.R. Elliot, Phys. Rev. Lett. 67, 711 (1991). A critical discussion regarding the physical soundness of such a peak was given some time ago by S. Veprek and H.U. Beyeler, Philos. Mag. B 44, 557 (1981).

${ }^{4}$ F.J. Bermejo, M.García-Hernandez, F.J. Mompeán, D. MacMorrow, and J.L. Martinez, Phys. Rev. B 51, 11932 (1995); see also, F.J. Bermejo, M. García-Hernandez, T. Mason, J.L. Martinez, E. Enciso, and A. Criado, ibid. 50, 13286 (1995).

${ }^{5}$ C. Condat and J. Jäckle, Phys. Rev. B 46, 8154 (1992); 52, 6238 (1995). For an application see K. Bhattacharya, U. Buchenau, and K.W. Kehr, ibid. 49, 8696 (1994).

${ }^{6}$ See for instance, S. Susman, K.J. Volin, D.G. Montague, and D.L. Price, Phys. Rev. B 43, 11076 (1991); B. Graneli and U. Dahlborg, J. Non-Cryst. Solids 109, 295 (1989).

${ }^{7}$ L.E. Busse, Phys. Rev. B 29, 3639 (1984).

${ }^{8}$ H.R. Glyde and E.C. Svensson, in Methods of Experimental Physics, edited by D.L. Price and K. Sköld (Academic, San Diego, 1987), Vol. 23B, p. 362. The $g(r)$ pair correlation of liquid ${ }^{4} \mathrm{He}$ evidences rather contrasting behaviors at both sides of the $\lambda$ transition. Within the superfluid phase the height of the main peak increases with temperature, whereas the opposite is found in the normal-liquid phase. Such a finding becomes entirely explainable by the increase of the condensate fraction as the temperature is decreased within the superfluid phase which makes the fraction of atoms with zero momentum increase. Such atoms because of the uncertainty principle are completely delocalized and therefore do not contribute to the intensity of $g(r)$.

${ }^{9}$ P. Vashista, R.K. Kalia, and I. Ebbsjö, Phys. Rev. B 39, 6034 (1989).

${ }^{10}$ M. Misawa, J. Non-Cryst. Solids 122, 33 (1990).

${ }^{11}$ A study on the effect of different fictive temperatures on the x-ray-diffraction pattern is given by Th. Gerber, B. Himmel, and J. Weigelt, J. Non-Cryst. Solids 126, 35 (1990). Some arguments in support of the existence of a large fraction of planar boroxol rings (i.e. up to $80 \%$ of boron atoms participating in such structures) are given by A.C. Hannon, D.I. Grimley, R.A. Hulme, A.C. Wright, and R.N. Sinclair, J. Non-Cryst. Solids 177, 299 (1994), whereas reconstructions of the experimental $S(Q)$ diffraction pattern by means of reverse Monte Carlo techniques, seem to point to a rather low portion of these structures. J. Swenson and L. Björjesson (private communication).

${ }^{12}$ G.K. White, S.J. Collocott, and J.S. Cook, Phys. Rev. B 29, 4778 (1984).

${ }^{13}$ S. Rau, Ch. Enss, S. Hunklinger, P. Neu, and A. Würger, Phys. Rev. B 52, 7179 (1995). Brillouin-scattering data at low (3-300 K) temperatures were reported by J. Pelous, Phys. Lett. 74A, 275 (1979).

${ }^{14}$ M.A. Ramos, S. Vieira, and J.M. Calleja, Solid State Commun. 64, 455 (1987).

${ }^{15}$ R.L. McGreevy and M.A. Howie (unpublished).

${ }^{16}$ R. Fernández-Perea, F.J. Bermejo, and E. Enciso, Phys. Rev. B 53, 6215 (1996).

${ }^{17}$ P.A.V. Johnson, A.C. Wright, and R.N. Sinclair, J. Non-Cryst. Solids 50, 281 (1982).

${ }^{18}$ A.C. Hannon (private communication). Data measured for several 
different temperatures show a consistent increase in the density of states at frequencies below some $20 \mathrm{meV}$.

${ }^{19}$ For an application of such a procedure see, for instance, M. Alvarez et al.., Mol. Phys. 66, 397 (1989).

${ }^{20}$ A.H. Verhoef and H.W. den Hartog, J. Non-Cryst. Solids 146, 267 (1992).

${ }^{21}$ P. Bruesch, Phonons: Theory and Experiments II, Springer Series in Solid State Sciences, Vol. 65 (Springer, Berlin, 1986), p. 142.

${ }^{22}$ B.T.M. Willis and A.W. Prior, Thermal Vibrations in Crystallography (Cambridge University Press, Cambridge, England, 1975), p. 142. Also, see, M.A. Krivoglaz, X-ray and Neutron Diffraction in Nonideal Crystals (Springer, Berlin, 1996), p. 182.

${ }^{23}$ See, for instance, U. Buchenau, J. Mol. Struct. 296, 275 (1993). According to the predictions of the "soft-potential model," a quasielastic broadening due to "soft modes" should exist, although such broadening only becomes evident at temperatures somewhat above $\approx 0.6 T_{g}$. For some detailed predictions of the soft-potential model, see, V.L. Gurevich, D.A. Parshin, J. Pelous, and H.R. Schober, Phys. Rev. B 48, 16318 (1993) and references to previous works.

${ }^{24}$ J.P. Bouchaud and A. Georges, Phys. Rep. 195, 127 (1990).

${ }^{25}$ See, F.J. Bermejo, F. Batallan, E. Enciso, R.P. White, A.J. Dianoux, and W.S. Howells, J. Phys. Condens. Matter 2, 1301 (1990). The Eq. (8) can also be obtained as a limit of the general expression due to K.S. Singwi and A. Sjolander, Phys. Rev. 119, 863 (1960).

${ }^{26}$ See, for instance, S.R. Nagel, Phys. Rev. B 16, 1694 (1977).

${ }^{27} \mathrm{Ph}$. Mangin, C. Tete, and G. Marchal, Solid State Commun. 50, 1073 (1984).

${ }^{28}$ See, for instance, W. Götze, in Liquids, Freezing and the Glass Transition, Proceedings of the Les Houches Session LI, edited by J.P. Hansen et al. (NorthHolland, Amsterdam, 1991), p. 289. 